\title{
Motor Unit Action Potential Duration: Measurement and Significance
}

\author{
Ignacio Rodríguez-Carreño, Luis Gila-Useros and Armando Malanda-Trigueros
}

Additional information is available at the end of the chapter

http://dx.doi.org/10.5772/50265

\section{Introduction}

The quantification of the bioelectric phenomena originating in nervous and muscular tissues is an essential task in diagnosis within the field of Electromedicine. Clinical electromyography is the part of Clinical Neurophysiology focused on the neuromuscular system, and includes the study of the electrical activity of peripheral nerves (electroneurography), striated muscles (electromyography, in its strict sense) and a number of reflex circuits (reflexology), among others [1].

The background to all these scientific areas is based on the parameterization of the bioelectrical functions of the neuromuscular structures. The definition and formulation of such parameters represents the theoretical and practical basis which enables the analysis of the function of muscles and nerves in normal and pathological conditions. The quantification of bioelectrical parameters makes possible to delimit their normal ranges. The presence of parameter values beyond normal ranges, as measured by neurophysiologic techniques, is used in the diagnosis of diseases of nerves and muscles, which is the main goal of clinical electromyography [2].

A basic concept in electromyography is the so called motor unit (MU), which represents the anatomical and functional element of the neuromuscular system. The MU is formed by the alpha spinal motorneuron and its innervated set of muscular cells. The electrical changes generated by activity of the MU can be acquired and amplified by electrodes located in muscle mass and these changes can be recorded and edited using electromyographic (EMG) devices. The representation of the changes generated by a MU is the so called motor unit action potential (MUAP). A MUAP waveform can be characterized by a number of parameters related to certain aspects of the structure and physiology of the MU (Figure 1). Therefore, the quantitative measurement of such parameters is a basic issue in electromyography, and the duration of the MUAP is a key measure as it defines the 
boundaries of the MUAP waveform and the rest of the MUAP parameters are measured within the time span defined by the MUAP duration [3].

The main parameters defined to characterize the MUAP waveform are reviewed in this chapter, which also covers the particular issues related to the significance and measurement of the MUAP duration.

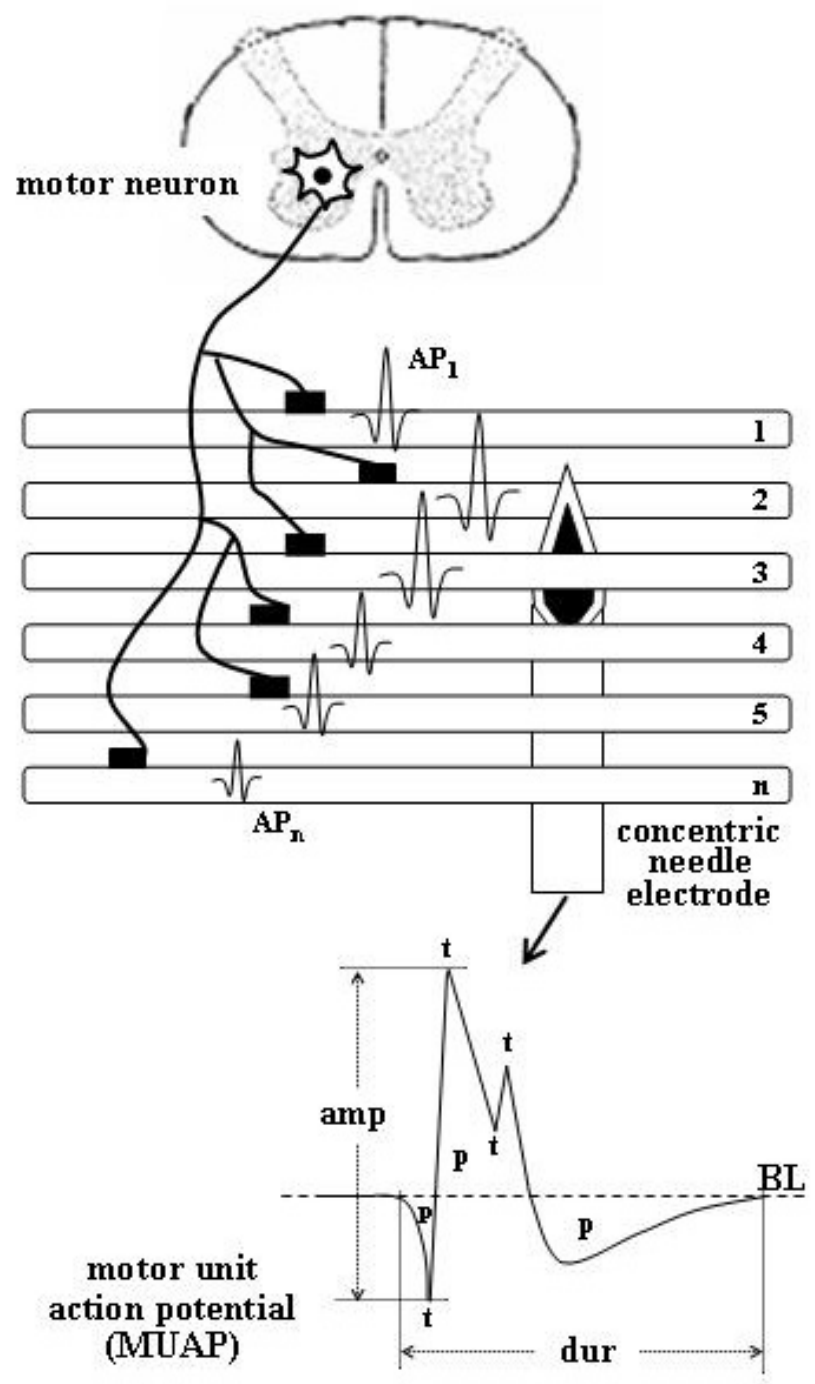

Figure 1. Schematic representation of a motor unit with $\mathrm{n}$ muscle fibers. The algebraic summation of the action potentials (AP) of all the single fibers present in the recording uptake area of the electrode $(\mathrm{AP} 1+\mathrm{AP} 2+\ldots+\mathrm{APn})$ generates the motor unit action potential (MUAP). The main parameters of the MUAP waveform are indicated: $a m p=$ amplitude; dur $=$ duration; $\mathrm{p}=$ phase; $\mathrm{t}=$ turn. $\mathrm{BL}=$ baseline. 


\section{Quantitative characterization of MUAP}

\subsection{Anatomical and physiological description of MU and MUAP}

Striated or skeletal muscles are the effectors of voluntary movements. Striated muscle cells or muscle fibers (MF) have an elongated form, and their contraction is brought about by the sliding of contractile protein filaments contained in their cytoplasm (sarcoplasm). As in any living cell, across the membrane of MFs there exists a difference of electric potential of approximately $90 \mathrm{mV}$ (the inside of the cell being negative with respect to the outside) because there is a difference in the amount of electrical charge in intra- and extra-cellular fluids. A basic property of MFs and neurons is the ability to change the membrane potential and transiently convert their inside into a positive potential in specific conditions. This inversion of potential or depolarization is called an action potential (AP), and it arises by a brief opening of membrane sodium channels, with the consequent rise in the membrane permeability to this ion. The changes of ionic fluxes related to the AP are transmitted towards neighbouring points of the membrane, being conducted along the MF at a velocity of $3-5 \mathrm{~m} / \mathrm{s}$. After the depolarization begins, the repolarization phase proceeds, in which there is further passive and active (by the action of the Na-K pump) transmembrane flux of ions, which restores the basal conditions of the membrane at rest. [4].

The nervous system controls the degree of contraction of the MFs by means of the frequency of the nervous impulses of the alpha motor neurons, whose central cellular components are located in the anterior horns of the spinal cord. These nervous impulses are APs of the motor neurons; they travel along the axons and are transmitted to MFs at neuromuscular junctions. As previously described, the system formed by an alpha motor neuron and its set of innervated MFs forms a MU, which represents the anatomical and functional unit of skeletal muscle. The number of MFs innervated by the MU varies according to the muscle. The number is small in the eye muscles, that need very precise adjustments; the large muscles of the lower extremities have several hundred MFs [5]. During a slight voluntary contraction, only a few MUs are activated, and they discharge APs at low frequencies (around 5 per second). To increase the strength of contraction, the nervous system drives a progressive increase in the discharge frequency and a progressive activation or recruitment of other MUs in the muscle concerned.

The recording and analysis of the electrical activity of MFs and MUs (myoelectrical activity) is the subject of electromyography. Conventional EMG studies are performed with needle electrodes that capture the activity of MFs within a hemisphere of $2.5 \mathrm{~mm}$ radius from the tip of the needle electrode. To study the MUAPs of a certain muscle, a needle electrode is inserted into the muscle mass, which the subject is asked to maintain under slight contraction. In this way, a low number of MUs are activated and the successive discharges of the corresponding MUAPs can be collected. If the degree of contraction is excessive, too many MUs are discharging and the recorded waveforms of their MUAPs are distorted by their superposition. 
In Europe, the needle electrodes currently used are concentric, which have a core of platinum or stainless steel embedded in insulating material located inside a stainless steel cannula. The core is the active electrode, and the cannula is the reference electrode. A MUAP is a recording of the changes produced by the discharge of the MFs of a MU (Figure 1). In general, normal MUAPs show mean peak-to-peak amplitudes of around $0.5 \mathrm{mV}$ and a duration from 8 to $14 \mathrm{~ms}$, depending on the size of the MUs. The size and shape of MUAPs is determined by certain structural and functional aspects of MUs. Pathologic processes of the peripheral nervous system (neurogenic processes) and of muscles (myopathic pathologies) can alter these aspects, leading to abnormal deviations in MUAP parameters; i.e., the EMG signal captures pathologic remodelling of the MUs caused by neuromuscular diseases. Once other neurophysiologic data and the clinical context of the patient have been taken into account, a deviation with respect to the normal pattern for a given muscle constitutes the basis of an EMG diagnosis.

\subsection{Parameters of the MUAP and their physiological significance}

To characterize a MUAP waveform quantitatively, a number of parameters have been defined (Figure 1). These parameters are related to certain anatomical and physiological aspects of MFs and MUs. There are three groups of MUAP parameters to characterize the size, shape and stability, respectively, of the MUAP. These parameters, which provide information about certain spatial and temporal characteristics of MF and MU activity, are described below:

1. Size parameters are related to the size (diameter), number and density of generators of a MUAP (i.e. the MFs of the MU). These parameters include duration, amplitude, area and indices such as the size index and thickness index. Since duration will be treated extensively later in this chapter, it will not be described in this section, where a brief description of the other parameters is given.

The amplitude is the voltage difference from minimum to maximum peaks. Computer simulations of MUAPs show that the amplitude is determined by the few MFs (less than eight) located within a semicircular uptake area of $0.5 \mathrm{~mm}$ radius from the electrode [6]. Consequently, amplitude can vary considerably within the MU territory (the space within which the MFs of a MU are randomly scattered).

Area can be calculated automatically by integrating the rectified MUAP within the duration. It depends on the MFs present within $1.5 \mathrm{~mm}$ from the core of the concentric electrode [7]. Relatively small movements of the recording electrode affect the amplitude and area parameters considerably because the amplitude of the APs of the MFs decays quickly with distance to the electrode [8].

In the quest for more stable estimators of the magnitude of MU generators, new parameters have been defined, the most relevant being the thickness and size indices. The thickness index is computed as the area-to-amplitude ratio, and is a sensitive detector of myopathic abnormalities [9], but not of neurogenic ones. To improve detection of neurogenic MUAPs, 
multivariate analysis was used to find the optimal separation from normal MUAPs; in this way, the size index was formulated as [2 $x \log _{10}$ (amplitude) + (area/amplitude)]. However, this index is not significantly better than other parameters for the detection of abnormality in myopathic conditions [10].

2. MUAP waveform shape parameters transcribe the temporal synchrony / dispersion of the activation times of the MFs and their conduction velocities. These parameters include the number of phases, the number of turns, and indices such as the coefficient of irregularity.

A phase is the part of a MUAP that falls between two baseline (BL) crossings. A turn is a peak (i.e. a point of directional change) in a MUAP waveform. The number of phases is counted within the MUAP duration. Various amplitude and duration criteria are used in computerized measurements to exclude from the count brief BL crossings or small peaks, which may be due to noise [11]. Normal MUAPs have simple shapes between two and four phases. Polyphasic MUAPs have more than four phases, and those with more than five turns are called polyturn or complex MUAPs. These terms all reflect the same feature: increased temporal dispersion of MFs potentials, but polyphasia indicates more pronounced changes.

To enhance the sensitivity and precision of measurement of MF synchronicity, other estimators have been proposed, such as the coefficient of irregularity [12]. This is defined as the total amplitude change (over the MUAP length) divided by the peak-to-peak amplitude. The minimum value that MUAP irregularity can have is 2 . As the complexity of a waveform increases, the value of this index increases too. Significant differences have been found between pathologies (neurogenic as well as myopathic) of both slow and quick progression [13]; but in general, and in spite of its theoretical background, the coefficient of irregularity has not shown better performance than conventional parameters.

3. Stability parameters or jiggle parameters have been defined to quantify the degree of variability in MUAP shape at consecutive discharges [14]. These parameters are the consecutive amplitude differences (CAD) and cross-correlational coefficients of consecutive discharges (CCC). The efficiency of CAD and CCC has been proved mainly in simulated signals. There are very few studies with real EMG recordings [15], but the presence of noise has been found to significantly affect quantification of the jiggle using these parameters, and consequently the estimation of jiggle still requires subjective verification by visual assessment.

\section{MUAP duration}

\subsection{Definition}

MUAP duration is defined as the time from the start of activation of MU fibers until the end of their repolarization phase, i.e., the time in which the bioelectric changes produced by a discharge of a MU take place. 


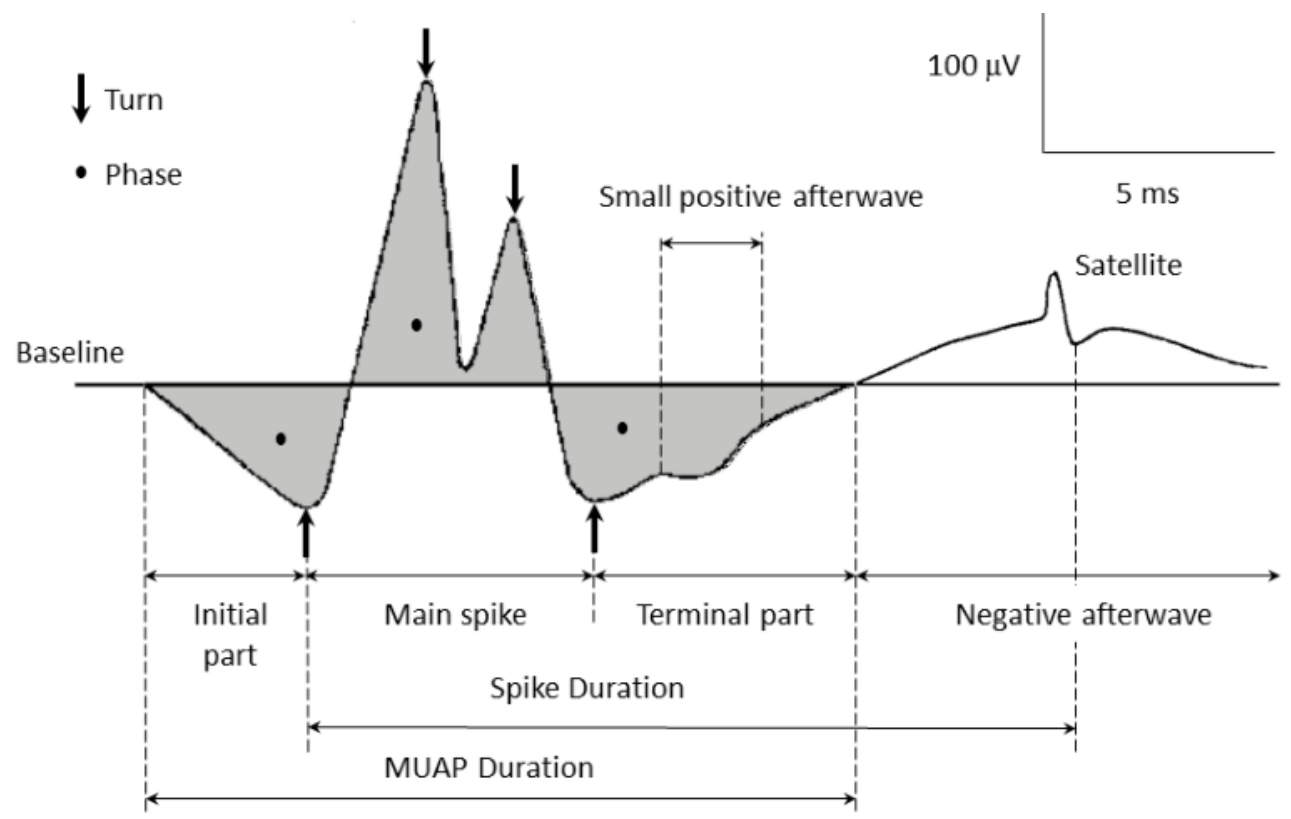

Figure 2. Parts of the MUAP. MUAP parameters: duration, spike duration, turns, phases.

\subsection{Parts of the MUAP}

Over the duration of a MUAP, several parts of the MUAP waveform can be delimited (Figure 2), each one having specific structural and functional significance [16]:

1. The initial part: from the start of MF activation to the first positive turn. Graphically this is a positive deflection whose charactersitics depend on the distance of the motor end-plate region until the situation of the recording electrode in the length of the fiber. If the electrode is close to the end-plate zone, the initial positive part in the MUAP hardly exists, and the MUAP waveform begins with an initial upward defection (Figure 3). As the distance between the end-plate region and the tip of the electrode increases, the initial part becomes more and more evident and its duration increases as well, being maximal when the electrode is located near the extreme of the MFs near the tendon. [3, 16-18].

2. The spike part: between the first and the last positive turns. The spike part mainly depends on the temporal dispersion of the MF potentials as they pass in the vicinity of the recording electrode. It is thought likely that only less than 15 fibers contribute to the spike part in normal MUs [19]. The spike usually has one negative peak, called the main spike, but may have several positive peaks. Note that a MUAP may contain spike components other than the main spike. Such parts are called satellites. Spike duration is measured between the first and the last positive peak of the MUAP (Figure 2). If the MUAP is recorded in the end-plate region, the start of the MUAP and of the spike part coincide, because there is no initial part. The spike duration is usually shorter than the 
total duration, but this does not need necessarily be so if a satellite is present. Satellites, which usually follow the terminal part (but exceptionally precede the initial part), are included in the measurement of spike duration and thus, spike duration may exceed the total duration of the MUAP [20].
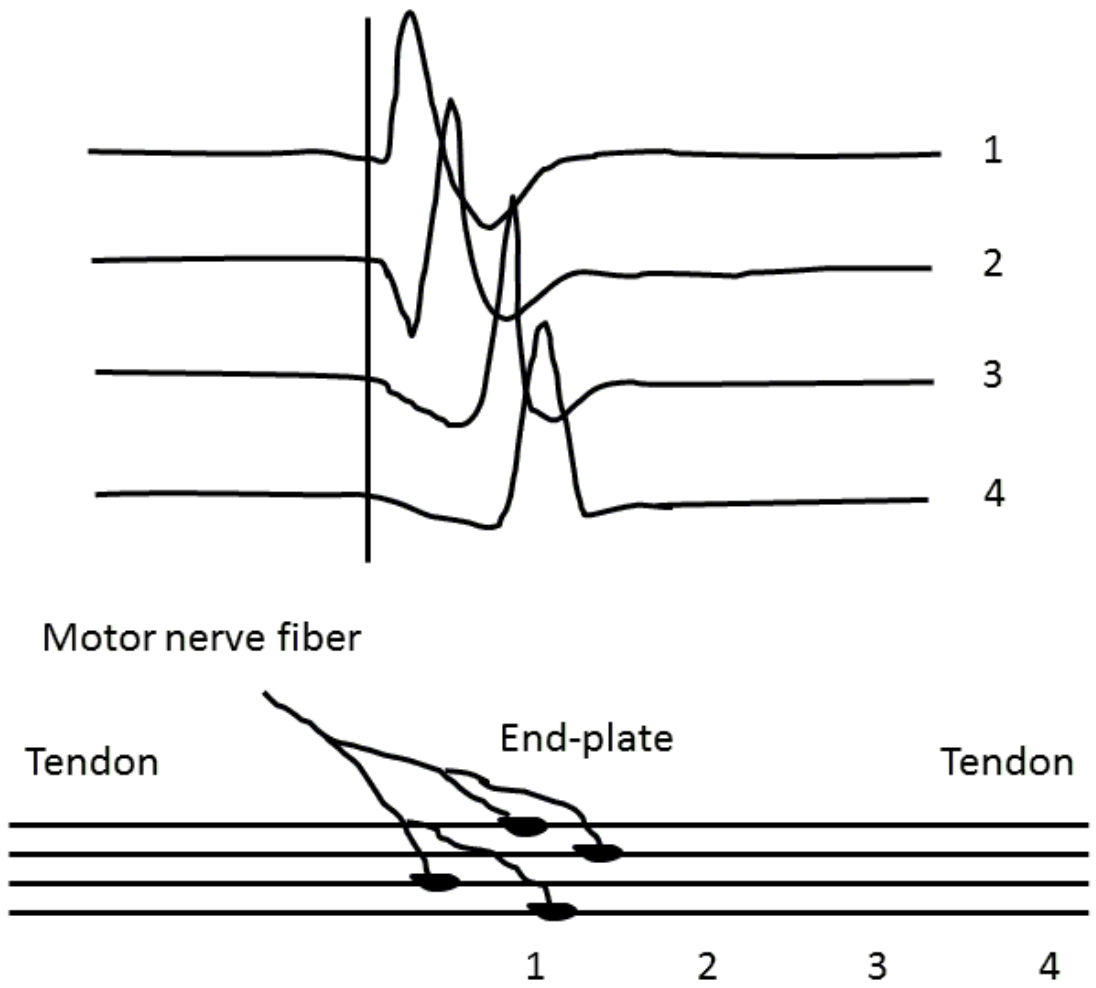

Figure 3. Example of recording a MUAP at different distances from the end-plate zone (position 1). As the distance from the end plate increases (1 to 4), the initial positive part of the MUAP becomes longer. Below, a schematic presentation of the recording positions with respect to the end-plate zone.

3. The terminal part: from the last positive turn until the endpoint, where the signal reaches the BL. The terminal part is longer than the previous parts because the approach to the BL is gradual. The terminal part is generated by the volley of APs leaving the electrode and it includes the main part of the repolarization phase.

4. Small positive afterwave: these are not usually seen in recordings with concentric electrodes, but can be observed within the terminal part of MUAPs in recordings performed with monopolar electrodes (which are more commonly used in the United States than Europe). The small positive afterwave reflects the arrival of MF depolarization at the muscle-tendon union with the tendon [21]. When a small positive wave is present, usually superimposed on the terminal part, it is included in the MUAP duration. 
5. Negative afterwave: this is an artifactual wave that arises due to the effect of the highpass filter of the amplifier (Figure 4), mainly when the MUAP has a dominating positive phase, which is counterbalanced by a negative afterwave [22, 23]. A negative afterwave usually has low amplitude (less than 10 microvolts), but, in any case, it is an artifact and should be excluded from duration measurements.
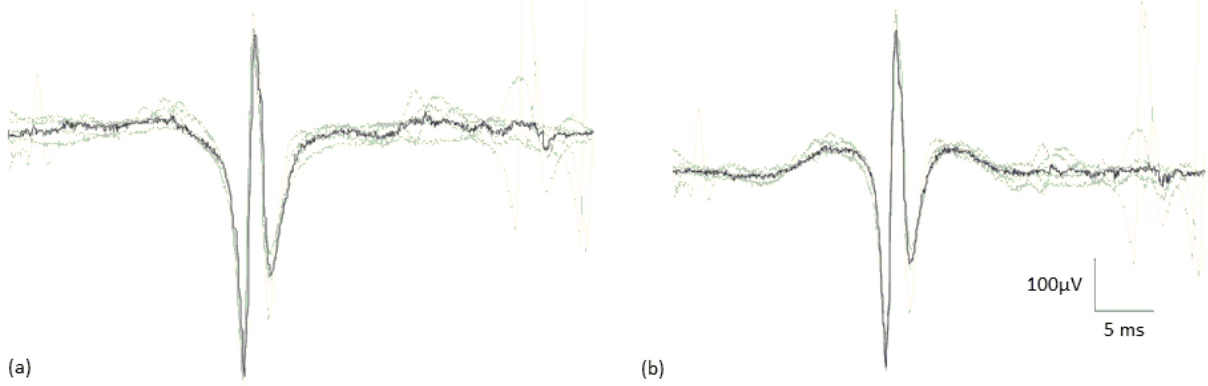

Figure 4. Effects of the high pass filter in the MUAP waveform. The cut-off frequencies of the filters 8 applied in (a) and (b) are 10 and $50 \mathrm{~Hz}$, respectively.

\subsection{Physiopathological significance of MUAP duration}

Computer simulations of the MUAP indicate that the duration reflects the current generated by the MFs within $2.5 \mathrm{~mm}$ of the active recording surface of the electrode [6]. The total current is determined by the number of MFs and their cross-sectional area. Duration is not affected by slight changes in the electrode position, in comparison to amplitude and area, both of which are sensitive to this change.

The total MUAP duration comprises the slow initial and terminal phases of the MUAP signal. These parts represent the time when the APs of the MFs are at some distance from the electrode and the APs are still relatively equidistant from the recording surface and contribute to a similar extent. Therefore, the duration of the normal MUAP is not so much dependent on the temporal dispersion of the individual MF APs but more on the number of MFs within the recording area [3]. Although the degree of temporal dispersion of the APs of MFs is specifically expressed by the spike duration and shape parameters, temporal dispersion also influences the magnitude of the total MUAP duration, as can be seen in pathologic MUAPs. When there is large variability in MF diameters, an enlarged end-plate region or a mixture of slow- and fast-conducting terminal axons, the temporal dispersion of MF potentials is pronounced, resulting in MUAPs with long durations and more or less complex waveforms (sometimes extremely complex).

The physiopathological correlations underlying the magnitude of total MUAP duration, makes the duration measurement clinically useful (Table 1). The duration is a parameter currently used in clinical electromyography and its normative values have been established 
over samples of normal subjects for each muscle and age range. [24, 25]. Reference values from healthy subjects show little correlation to gender, height and weight. Within the age range of 15 to 65 years the effect of age is negligible [26], but an increase of duration has been reported for subjects of older ages [27].

\begin{tabular}{ll}
\hline MUAP abnormality & Anatomical phenomena related \\
\hline Decreased amplitude & $\begin{array}{l}\text { Muscle fibers' atrophia } \\
\text { Increasement of connective tissue } \\
\text { Excessive jitter and blocking }\end{array}$ \\
\hline Increased amplitude & $\begin{array}{l}\text { Muscle fibers grouping (reinervation, regeneration) } \\
\text { Muscle fibers hypertrophia }\end{array}$ \\
\hline Decreased duration & Muscle fibers' atrophia \\
& Loss of muscle fibers \\
& Serious MUAPs blocking in endplate \\
\hline Increased duration & Increase in the number of muscle fibers (collateral \\
& growing) \\
\hline Increased spike duration & Variation in the diameter of the muscle fibers \\
& Increase in the width of the endplate \\
\hline Increase in the number of turns and & Slow conduction in terminal axons \\
phases & Increase in the width of the endplate \\
& Increase in the variability of the diameter of muscle \\
& fibers \\
\hline Increase in the firing rate & Loss of MUs \\
& Decrease in the force generated by individual MUs \\
\hline Increase in the jiggle & Abnormal neuromuscular transmission \\
\hline
\end{tabular}

Table 1. Relation between MUAP alterations and abnormality reflected.

For the EMG examination of a muscle, a sample of 20 MUAPs must be extracted [18]. The mean values of the different MUAP parameters are matched up with their respective reference values. Deviations from normality may be defined as a value of mean duration plus/minus 2 standard deviations above or below that for samples from the normal population for the same muscle and age group as the subject under study [28].

Abnormally high duration values result from an abnormal increase in the number of MFs in the MUs in neurogenic processes due to collateral reinnervation and focal grouping. The neurogenic MUAPs can have simple or complex shapes and can be stable or instable (normal or increased jiggle) depending on the nature of the pathology and its temporal course (acute, subacute or chronic). With regard to abnormally low duration values, a low duration reflects loss of MFs in myopathic processes, myophatic atrophy of MFs or neurogenic lesions at early stages of reinnervation (nascent MUAPs), or severe blocking of neuromuscular transmission (such as in botulism or myasthenia gravis).

MUAP duration is a basic parameter of the MUAP due to its physiopathologic significance and also due to the fact that the duration markers (the established start and end points) 
define the boundaries of the MUAP waveform and thereby separate those parts of the recorded signal which will be analyzed from other parts, such as BL or background activity. All MUAP parameters and features are measured within the MUAP duration or, in the event of the presence of satellites, with respect to it; consequently, duration is the first parameter that must be determined.

\section{Measurement of MUAP duration}

\subsection{A challenge for quantitative electromyography}

Technical improvements implemented on recent EMG machines have made many aspects of EMG examinations easier. Examples of such improvements are facilities for extraction of MUAP signals; edition, storage, automatic measurement of parameters; calculation of mean values; and the process of matching normative ranges. However, in clinical electromyography, diagnostic judgment, i.e., the final diagnostic conclusions built upon the collected data, is still mainly dependent on the knowledge and experience of the electromyographist who performs the study. Quantitative methods try to overcome subjective considerations by means of precise measurements of physiopathologically significant features. The performance of such methodologies is in general satisfactory when the conditions of the study are favorable: a collaborating patient, a fully developed pathology, and low levels of noise. But, working circumstances are seldom so perfect, and there are still important limitations mainly due to two disrupting factors that currently can only be partially controlled: variability and noise. In this respect, the measurement of MUAP duration can serve as a paradigmatic example of a fundamental challenge facing clinical neurophysiology: how to extract objective and consistent parameter estimates. The nature of the challenge is shown in the following considerations.

\subsection{Clinical and physiologic duration}

The definition of MUAP duration is, as stated above, simply the time between the beginning and the end of the bio-electrical activity of the MUs detected by the recording electrode. Often, the "duration onset" can be easily determined because the takeoff of the MUAP waveform, which is associated with the depolarization of MFs at the end-plates, is so abrupt that the waveform appears clearly deflected from the BL. This occurs especially if the recording has been made close to the end-plate zone and if the MUAP does not have an initial negative part. However, the "duration end", which is not associated with any clearly identified physiological event, is more difficult to determine because the terminal part of the waveform approaches the BL gradually. With real recordings and in simulation studies, it has been demonstrated that the extinction of APs continues for over $20 \mathrm{~ms}$ after the main spike of the MUAP [29-31]. In real recordings, a very stable BL and a large number of averaged discharges are needed in order to observe such a slow return to the BL in the terminal part of the MUAP. Routine recordings, however, almost invariably have slow BL fluctuations and other noisy interference that obscure the full extension of the terminal part. Thus, two meanings of "duration" should be considered: the "physiologic" (as defined 
above) and the "clinical" $[29,30]$. The above considerations are indicative of the operational difficulties encountered with the simple physiologic definition of MUAP duration. The concept of clinical duration is that applied generally in diagnostic applications and will be used in the rest of this text. As with physiologic duration, there are difficulties in the measurement of MUAP clinical duration. These difficulties are discussed below.

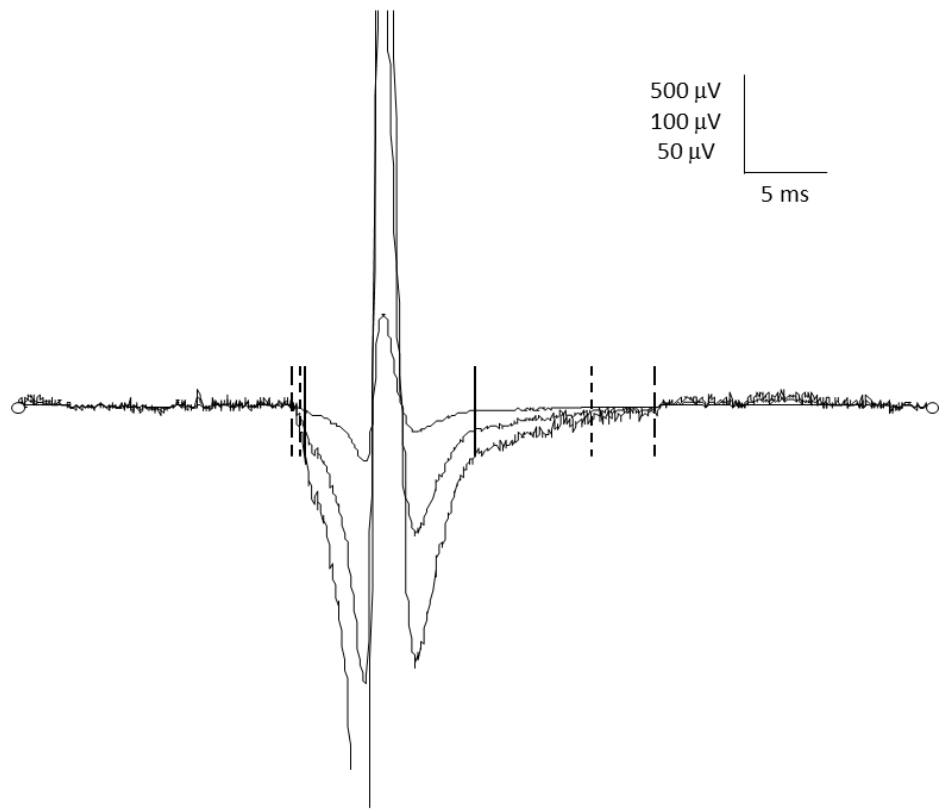

Figure 5. The same MUAP displayed at different gains. As the MUAP is amplified, its duration is measured longer due to the visual effect. Continuous, short dashed, and dashed lines represent the duration markers at 500, 100 and $50 \mu \mathrm{V} / \mathrm{cm}$, respectively.

\subsection{Manual measurement of clinical MUAP duration}

Clinical MUAP duration is defined as the time between the start and end points of the

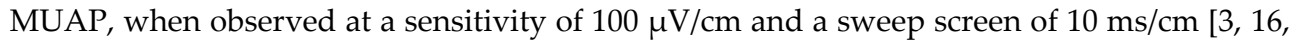
29]. At higher gains, duration measurements tend to be longer because more of the slight initial or terminal slopes are visible before they merge with the random noise of background activity [23], see Figure 5. The gain of $100 \mu \mathrm{V} / \mathrm{cm}$ was arbitrarily chosen to standardize the visual resolution at which duration markers should be manually placed. In this way, duration can be conceived of as a morphological feature, operationally defined in accordance with a specified magnitude of display resolution at which the recorded signal is represented.

When making manual measurements, electromyographists measure MUAP duration by visual inspection at the standardized settings stated above. Manual measurements can be made for an isolated discharge, over the averaged potential resulting from a set of MUAP 
discharges or by visual inspection of a set of discharges in superimposed and/or raster modes.

\subsection{Automatic measurement of MUAP duration}

A number of algorithmic methods for automatic measurement of duration have been designed and implemented on commercial equipment. Such algorithms aim to reproduce the manual procedure, and those used in computer-aided methods include BL calculation and use quantitative amplitude or slope criterion or a combination of both to look for the limit points between the MUAP waveform and the BL $[3,16]$. Quantitative definitions applied to the analysis of morphologic features of the MUAP are similar to the automatic counting of turns and phases [32,33]. Usually these algorithms are applied to the averaged MUAP waveform obtained from the discharges that have been recorded and extracted with automatic assistance [34, 35].

One might expect these algorithms to be more reliable than manual measurement, but in fact they suffer from several limitations when dealing with real signals. High variability has been observed in automatic as well as manual measurements. In addition, automatic measurements are often inaccurate, always require visual supervision, and frequently require manual correction of duration marker positions.

\section{Variability of manual measurements}

Duration has long been recognized as the most difficult MUAP parameter to define and measure in an unequivocal way, and exact positioning of the endpoint is recognized to be somewhat arbitrary [16]. It is therefore likely that the inter- and intra-examiner variability of manual duration measurements is greater than that for the other MUAP parameters. An important consequence of this variability is that the normal limits of MUAP duration for a given muscle and age range have broad margins, which drastically reduce the diagnostic sensitivity of the parameter [36]. Thus, whilst large deviations from normality are easily identified, the intepretation of the significance of smaller deviations depends considerably on the examiner.

Several studies have investigated the variability of repeated manual duration measurements. In one study, a set of 25 nearly-normal MUAPs recorded from the brachial biceps muscle were manually analyzed three times on different days by the same single electromyographist. In the three repeated manual measurements, the mean durations ranged from 14.9 to $15.7 \mathrm{~ms}$, and the largest difference between durations of MUAPs from the same MU was $8 \mathrm{~ms}$ [16]. Similar observations of such low degrees of reliability of manual duration readings have been reported by other authors [37-39].

In another study, for a systematic quantitative estimation of the intra- and inter-examiner variability in MUAP duration measurements, the Gage Reproducibility and Repeatability (Gage R\&R) method [40,41] was applied [42]. This method is based on the analysis of the 
variance of repeated measurements of a given feature, and it is currently applied in industrial quality control studies. It was designed to assess both the variability in product magnitudes caused by the production process itself (part-to-part variability) and the variability attributable to the measurement system (the gage). The latter component of variability includes that attributable to the measurement device (the repeatability or intraoperator variability), assessed by repeated measurements by the same operator, and that attributable to the operator (reproducibility or interoperator variability), assessed by comparison of the measurements made by different operators. In the context of MUAP duration measurement, the part-to-part variability is related to the intrinsic variability of MUAP duration present in each sample of MUAPs extracted from a given muscle. This intrinsic variability of MUAP duration (i.e. variability of the object being measured as opposed to the process of measurement) is due to differences in size and structure of a muscle's MUs and to differences in electrode positioning within the muscle.

The Gage R\&R method was applied to six independent duration measurements performed by two electromyographists (three measurements separated in time by each electromyographist) on a set of 240 MUAPs from two muscles without pathology: the tibialis anterior and the first dorsal interosseous. The MUAPs accepted for analysis had well-defined waveforms and were free of superposition, gross BL fluctuations and distortions of other sources. In order to make manual measurements, an interactive software tool displaying the averaged MUAP and the set of the extracted discharges in raster and superimposed modes was provided. The time base and sensitivity could be changed by the operators, but the sensitivity and sweep speed for placing duration markers was fixed at the standard values of $100 \mu \mathrm{V} / \mathrm{cm}$ and $10 \mathrm{~ms} / \mathrm{cm}$, respectively.

In spite of the favourable conditions (the clean and well-defined MUAP waveforms and the good-quality visualization and measurement software), a high degree of variability in duration measurements was observed. Of the six evaluations of start marker position, the biggest difference for a MUAP was $6.6 \mathrm{~ms}$. Broader ranges, up to $11.2 \mathrm{~ms}$, were observed for end marker positions. The biggest ranges were observed in end marker positions for MUAPs with a long and gradually-sloped terminal part to their waveforms (Figure 6a). This particular feature of MUAP waveforms was found to be the major cause of difficulty in the manual procedure, since other confounding factors, such as the presence of noise, BL fluctuations and secondary MUAPs in the recordings, were minimised at the time of selecting samples of MUAPs for the study. Examples of other difficulties encountered in manual placement of duration markers are given in Figure 6.

The reproducibility and repeatability analysis by the Gage R\&R method decomposes the total variability of the measurements into that intrinsic to the sampled MUAPs (the part-topart variability, i.e., the variability in the measured parameter per se) and the variability attributable to the electromyographists. The latter component accounted for over $30 \%$ of total variability and was mainly due to variability in repeated measurements by the same examiner (intraoperator variability). In industrial contexts, where the Gage R\&R method is 
frequently used, degrees of operator variability greater than $10 \%$ are considered as poor, and greater than $30 \%$ as unacceptable [43].

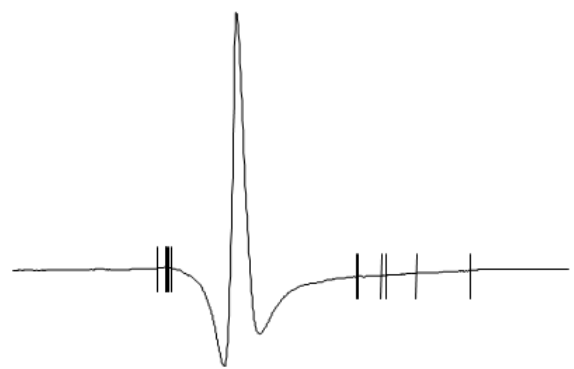

(a)

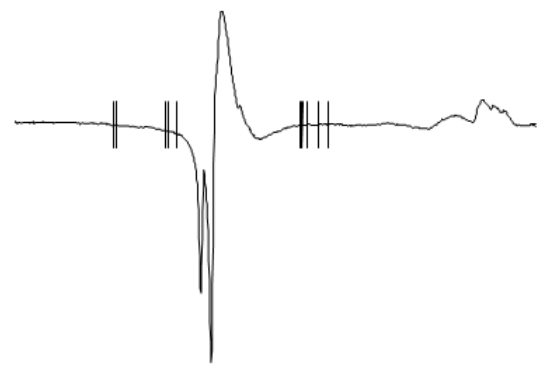

(c)

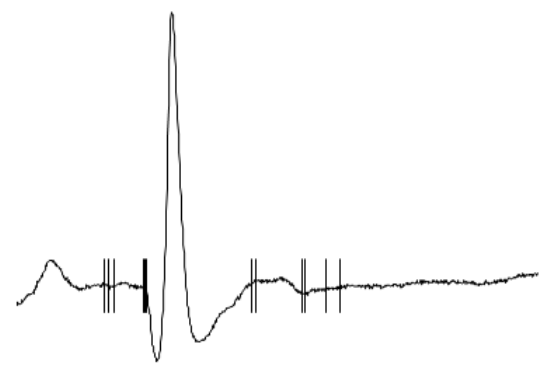

(b)

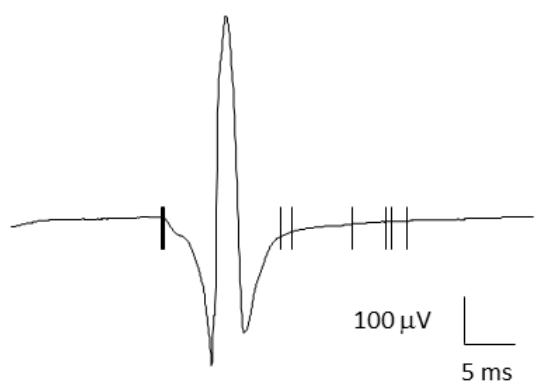

(d)

Figure 6. Variability in the manual placement of the duration markers. For different electromyographists, there are usually small differences in the manual positions of the start markers (a, d). Great dispersion in the position of the start or end marker can be seen occasionally in the initial part of the MUAP when it has a low slope $(\mathrm{a}, \mathrm{c})$. Superimposed discharges of other MUAPs over the initial or terminal portions of the MUAP waveform (b) and the presence of two different slopes separated by an inflexion point at the final portion of the MUAP (d) can be other sources of greta variability in the position of end marker.

\section{Proposal of a "gold standard" for MUAP duration measurement}

As can be concluded from the above discussion, a manual procedure does not guarantee consistent and reliable measurements of MUAP duration. Therefore, if duration markers are automatically placed by a modern EMG device and an error is detected by visual inspection, manual correction does not ensure an accurate estimate of MUAP duration.

In order to assess the effectiveness of a given automatic method of MUAP duration measurement, it is necessary to have available a "gold standard" of duration marker positions (GSP), that is, the marker positions which the automatic method should be finding automatically. Since, as a result of the conceptual and operational limitations exposed 
above, no single manual measurement can be accepted as the true and exact one, a probabilistic approach to the definition of the GSP has been proposed [42]. For the start or end point of a given MUAP, the GSP was calculated from a set of six marker positions obtained from the repeated marker placements made by two examiners. Specifically, the GSP was calculated as the mean of the three marker positions that were closest together.

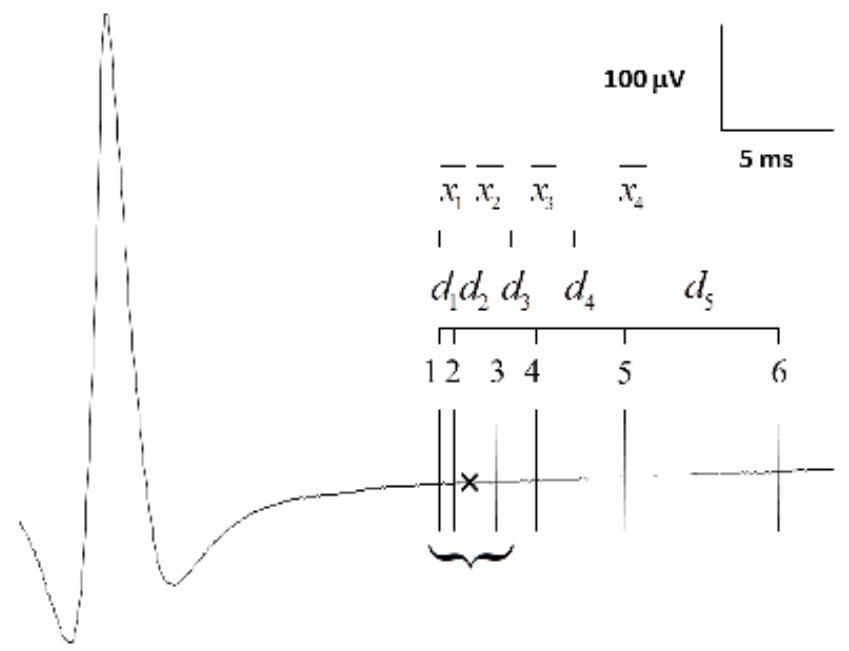

Figure 7. Determination of the gold standard of the GSP in an example of six manual marker positions of the end point.

As illustrated in the example in Figure 7, the six markers were ordered by their respective time values from lesser to greater ( 1 to 6$)$. The five differences between the six position values were obtained ( $d_{1}$ to $\left.d_{5}\right)$ and the means between two consecutive differences were calculated ( $\overline{x_{1}}$ to $\left.\overline{x_{4}}\right)$. The smallest of the four mean values was selected ( $\overline{x_{1}}$ in this example) and the GSP (marked with a cross in the figure) was obtained as the mean of the three manual markers with lowest mean difference (markers 1, 2 and 3 in this example) [42]. By means of this approximation, although a position cannot be assumed to be "true" or even "the best", it can be regarded as a "most likely" position. Thus, such a position can be adopted as a GSP on the basis that it is better in a probabilistic sense than any single position made by manual placement.

\section{Description of conventional methods for automatic measurement of MUAP duration}

The use of computer-aided measurements can theoretically resolve the problem of intraand inter-examiner variability. The execution of any algorithm on the same signal will always give the same results, without any variability in repeated measurements. In view of this, several automatic methods were developed to try to reproduce the manual procedure used by electromyographists, using amplitude and/or slope criteria to look for the limit 
points of the MUAP waveform with respect to the BL. Among the reported methods there are differences in several aspects, as described in detail in [16] and [35]. To illustrate these computer-aided techniques, a brief description of several conventional automatic methods (CAMs) is given below. Descriptions include a consideration of differences in the extraction procedure of the MUAP waveform, the definition of the $\mathrm{BL}$ and the criteria applied to find the MUAP start and end points (the duration markers positions). The five methods reviewed are the Turku method 1 (T1), the Turku method 2 (T2), the Uppsala method 2 (U2), the Aalborg method (AM) [16], and the Nandedkar's method (NM) [35].

The methods calculate the MUAP duration within a 40,50 or $100 \mathrm{~ms}$ long analysis window. MUAP waveform extraction procedure differ in the following ways:

- In T1 and T2, MUAPs are manually isolated with a trigger level. To reduce high frequency noise and the effect of the presence of other MUAPs in the analysis window, 100 discharges are averaged.

- In AM, MUAPs are automatically isolated and classified by a template matching method using the main spike of the potential. From the set of discharges of the same MUAP, the three most similar ones are selected to obtain the averaged waveform.

- In U2, MUAPs are manually isolated, and the MUAP waveform is obtained by averaging between 20 and 200 discharges.

- In NM, MUAPs are automatically isolated, identified and classified using a multiMUAP system. From 50 to 65 discharges are extracted for each MUAP, and the MUAP waveform is obtained using median averaging.

With respect to definition of the BL and the MUAP start and end markers, the different criteria used by the five automatic methods are outlined below:

- $\quad$ In T1 and T2, the BL is the average of samples at both 3 and $4 \mathrm{~ms}$ ends of the analysis window. NM calculates the BL as the average of the first $5 \mathrm{~ms}$. U2 and AM calculate the $\mathrm{BL}$ as the electrical zero.

- $\quad$ Once the BL has been subtracted, T1 and U2 begin their searches for the start and end of the MUAP from the start and end of the analysis window, respectively. T2 and AM begin their searches from a triggering point in the rising slope of the main spike. NM begins its search from the maximum peak.

- $\quad \mathrm{T} 1, \mathrm{~T} 2, \mathrm{AM}$ and U2 use thresholds related to the amplitude/slope values of individual samples or windows of samples (Figure 8a). NM uses thresholds related to the area under the MUAP and to the amplitude sample values (Figure 8b).

\section{Accuracy of conventional automatic methods}

Automatic measurements are free of the intra- and inter-examiner variability present in manual measurements. On ideal EMG signals with well-defined waveforms and without noise, the algorithms may perform satisfactorily. But on real recordings, the available methods for automatic measurement of MUAP duration demonstrate poor agreement and low stability [32]. Thus, visual inspection is always necessary and manual cursor adjustments are frequently required. This is the everyday experience in clinical practice; 
And it has been reported by various authors that manual correction of automatic placements is required for $20-50 \%$ of MUAPs [26, 34, 38, 44].

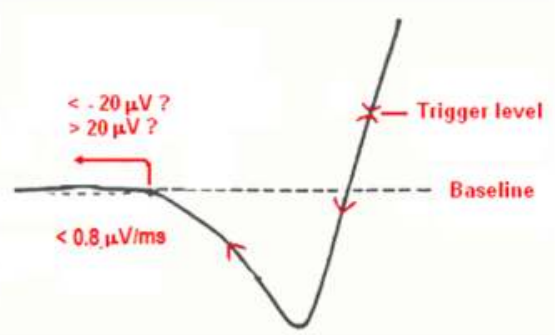

(a)

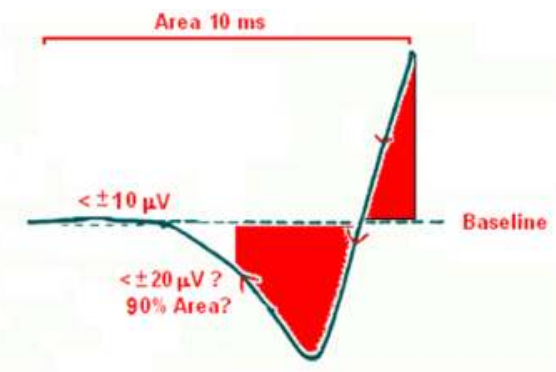

(b)

Figure 8. Description T2 and NM. In T2 (a), the MUAP onset is determined from the trigger to a point with slope $<0.8 \mu \mathrm{V} / \mathrm{ms}$ over a $1 \mathrm{~ms}$. If there is a point before with amplitude $>20 \mu \mathrm{V}$, a new point fulfilling the slope criterion is looked for. In NM (b), the peak with maximum deviation from the BL is identified. The area of the MUAP from the first sample to the peak is calculated. Then the sample point with $90 \%$ of this area to the peak is obtained. If the absolute amplitude at this point is greater than 20 $\mu \mathrm{V}$, a sample with $10 \mu \mathrm{V}$ amplitude towards the beginning of the window will be the MUAP onset. Otherwise a point toward the peak with $20 \mu \mathrm{V}$ amplitude is reached. The MUAP onset then will be the point with amplitude $10 \mu \mathrm{V}$ toward the first sample.

The accuracy of CAMs has been systematically assessed in normal and pathological MUAPs [42] [45]. Comparing the GSPs (determined by means of the probabilistic method referred above) with the marker positions obtained with CAMs (Figure 9), mean differences of up to $8.5 \mathrm{~ms}$ were found, with the T1 CAM. Absolute differences of more than 5 ms between the GSP and an automatic marker position (considered gross errors) were found in many cases: from $15.0 \%$ for AM end markers to $49.6 \%$ for U2 end markers.

In pathological MUAPs, the worst CAM results were observed with chronic neurogenic MUAPs, which have unusually long duration and are highly polyphasic (Figure 10c and 10d). The results were slightly better with myopathic (Figure 10a) and subacute neurogenic MUAPs (Figure 10b). Analysis of the mean and standard deviation of differences to the GSP (bias and precision, respectively) of the CAMs, showed that some methods, particularly the NM method, provided relatively good results with some pathologic MUAP groups. However, rates of gross errors (differences greater than $5 \mathrm{~ms}$ ) were seen in around $40 \%$ of estimates for several pathologic groups.

In general, end marker placement presents higher levels of error than start marker placement. As in the manual procedure, errors in end marker placement are more pronounced for MUAPs with long-tailed terminal parts. (Figure 10c). Other important sources of error that reduce the performance of CAMs are the presence of several kinds of noise in the recordings, such as the superposition of secondary MUAPs over the analyzed MUAP or BL, and BL fluctuations (Figure 9a. 9b, 9c). 


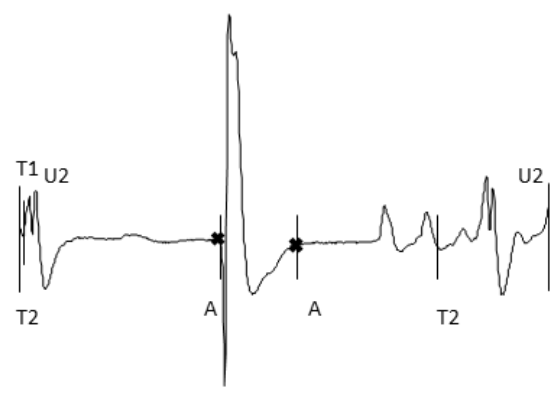

(a)

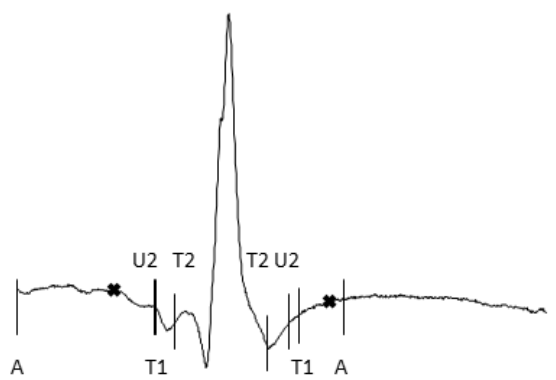

(c)

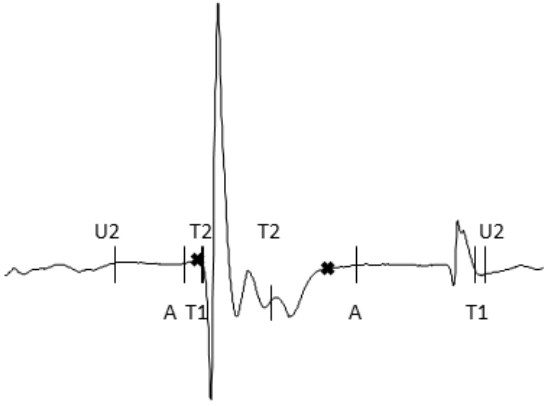

(b)

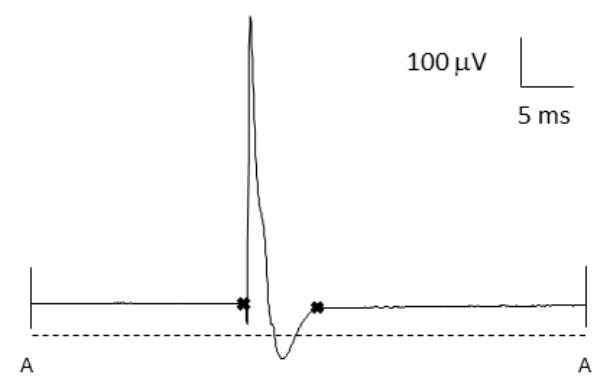

(d)

Figure 9. CAMs Errors in normal MUAPs. The presence of discharges of secondary MUAPs upon the BL before or after the analysed MUAP waveform induces gross errors in U2 and T1 ( $a$ and b).

Distortions of the MUAP waveform may cause errors in automatic placements (start markers in c). Poor agreement can be seen among the automatic end marker placements in MUAPs with relatively slow terminal slope (c). Misplacements can also result from an inadequate estimation of the BL, calculated as a constant value, electrical zero in Aalborg method (d).

An attempt to improve the performance of CAMs was carried out by means a signal process to accommodate BL fluctuation [46]. Conceptually, the EMG signal may be considered as an isoelectric BL (zero value) in which the discharges of the active MUs are superimposed. But in real recordings, the BL always shows slow fluctuations due to the activity of distant MUs and movements of the needle electrode. Two problems arise: on the one hand, the high-pass filter does not fully clean all the slow fluctuation and, on the other hand, if the high-pass filter's cut-off frequency is too high, the MUAP waveform can be distorted by creation of a more or less prominent negative afterwave, as previously described. The conventional approaches for dealing with the BL, are either to regard the BL as a straight line [23] of zero value (used by the $\mathrm{U} 2$ and AM methods) or to regard the BL as the average of the samples in initial and final segments of the analysis window (used by T1, T2 and NM methods) [16, 35]. An alternative approach for cancelling the BL fluctuation is to reconstruct the course of the BL followed by specific filtering designed not to distort the MUAP waveform [46]. For this 
purpose, standard methods as adaptive filters have been found unsatisfactory. The sequential application of several techniques of signal processing was necessary, including: 1) wavelet transforms for identifying segments of the EMG signal free of MUAP discharges, 2) averaging of the samples of each of these segments, 3) reconstruction of curves through the averaged points using cubic splines, 4) frequency analysis of this reconstructed BL, and 5) specific filtering based on autoregressive (AR) modeling. In spite of the sophisticated cancellation of BL fluctuation demonstrated by this method, the MUAP duration results of the five CAMs evaluated were not significantly improved when they were provided with signals that had been submitted to it [47]. To optimize automatic duration measurement, strategies other than, or in addition to, BL treatment are required.

(a)

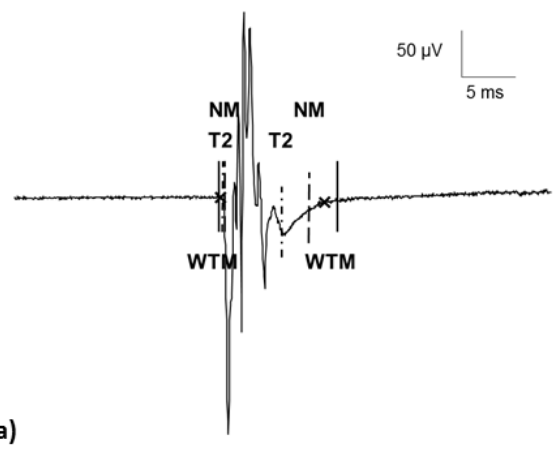

(c)

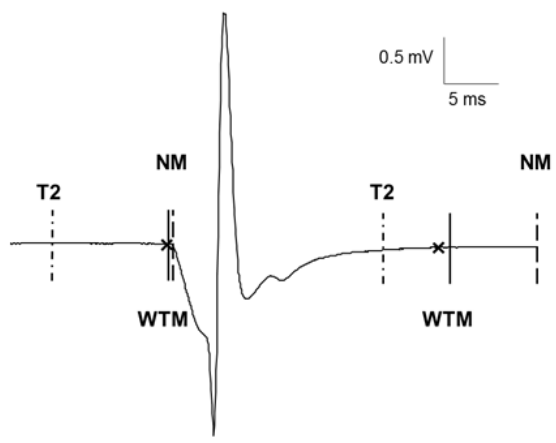

(b)

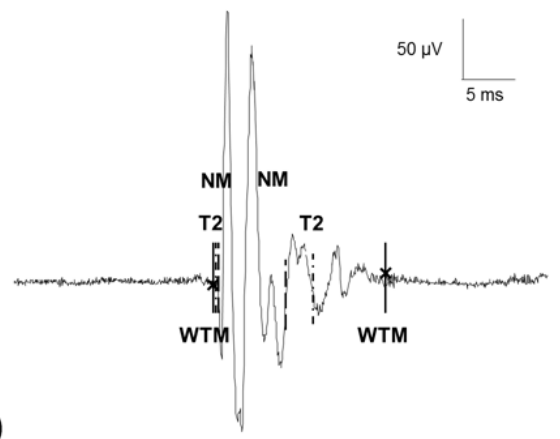

(d)

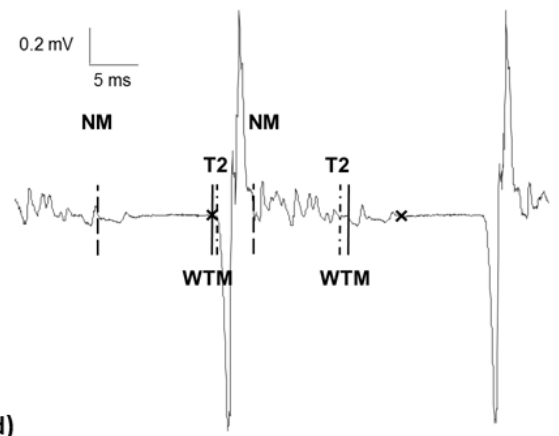

Figure 10. Performance of the CAMs with best results (T2 and NM) and the new duration method based on the wavelet transform (WTM) in pathological MUAPs: myopathic (a), subacute neurogenic (b), and chronic neurogenic MUAPs (c and d).

\section{New techniques of automatic measurement of MUAP duration}

The computational capacity of new computer systems enables the design and implementation of more complex algorithms for the automatic processing of the EMG recordings. Signal processing techniques such as the wavelet transform have been applied in the research and development of alternative automatic algorithms for measurement of MUAP duration. 
The discrete wavelet transform (DWT) is a technique that simultaneously obtains a time and a scale representation of signals and has been successfully applied for detecting biological events [48]. This technique has provided promising results in the analysis of various electrophysiological signals such as blink reflex [49], EMG and electrocardiographic recordings [50-52], electroencephalographic signals for analysis of epileptic activity [53], and event-related potentials [54]. By regarding transformed EMG signals at a suitable scale in the DWT domain, it is possible to evade high frequency noise and low frequency BL fluctuations. Thus the DWT provides a useful way to detect the boundaries between the MUAP waveform and the $\mathrm{BL}$, that is, for measuring MUAP duration.

A method based on the DWT was applied for measuring the MUAP duration [45, 55]. A schematic description of this method is given in Figure 11. The MUAP waveform consists of a set of peaks (Figure 11a) and the method makes use of the DWT with the non-orthogonal quadratic spline wavelet to detect not only the MUAP but also the start and end points of these peaks. The method selects two intermediate scales (one to find the start and another to find the end marker) from the DWT (Figure 11b) that represents the MUAP signal in terms of energy (thereby evading noise and BL fluctuation). In these DWT scales the peaks related to MUAP peaks are identified (Figure 11c) and amplitude and slope thresholds are used to determine MUAP start and end points (Figure. 11d). For finding MUAP start and end markers, this wavelet transform method (WTM) makes use of 10 parameters, which include the amplitude and slope thresholds. In the study, a genetic algorithm was applied to a sample of normal MUAPs in order to calculate the values of the WTM parameters [56].

This DWT-based automatic method was compared to other available algorithms and found to perform excellently, achieving accurate results for both normal and pathological MUAPs. Duration marker positions were significantly better than those of the other CAMs tested: the DWT-based method was the least biased and the most precise method as evidenced by the fact that it demonstrated the lowest mean and the lowest standard deviation of differences to the GSP. These improvements were observed with both normal and pathologic MUAPs, including myopathic, subacute and chronic neurogenic MUAPs, and also with fibrillation potentials $[45,55]$ (Figure 10).

The DWT-based method deals better with problems such as the presence of secondary MUAPs, BL fluctuations or high-frequency noise, performing equally well on signals recorded by various different commercial EMG hardware with varying amounts of technical noise. The rate of gross aberrant errors in start marker placement is low: $2.9,0.8$ or $0.0 \%$ for normal MUAPs, myopathic MUAPs and fibrillations, respectively. For the end marker, gross errors were more frequent: up to $27.6 \%$ for chronic neurogenic MUAPs, and around $10 \%$ for other kinds of pathologic MUAPs and for normal MUAPs. Although having less influence in the DWT-based method, the sources of error are the same as those for the other CAMs tested: long and high polyphasic waveforms (such as in chronic neurogenic MUAPs), the presence of consecutive peaks with low amplitude variation in initial or terminal parts (Figure 12a), and a low-sloped tail in the terminal part (Figure 12b). The latter is not detected by the DWT method because there is no corresponding maximum-minimum pair in the DWT. 


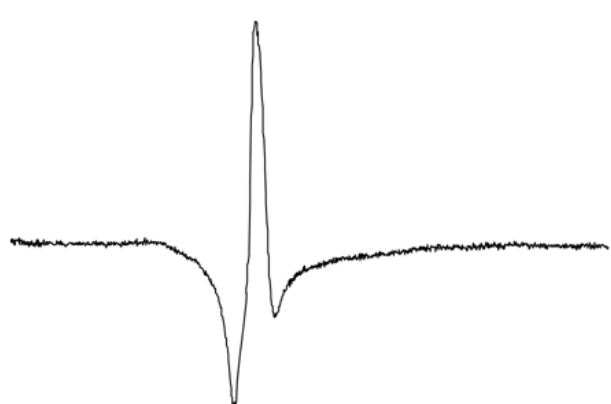

(a)

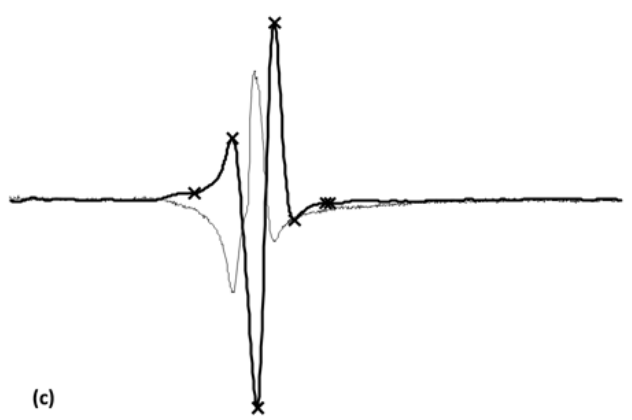

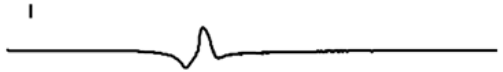

II

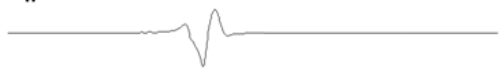

III

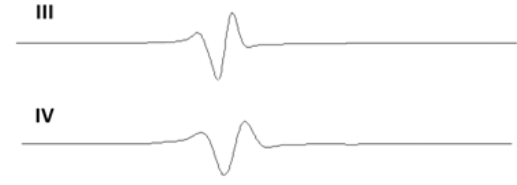

(b)

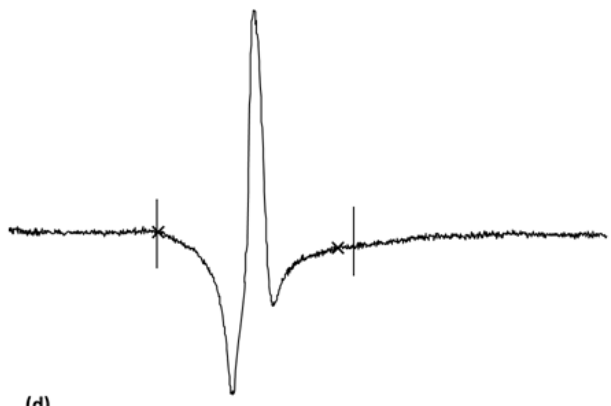

(d)

Figure 11. New method based on the DWT. (a) Original MUAP. (b) The MUAP (I) and the DWT at scales 4 (II), 5 (III) and 6 (IV). (c) MUAP and selected wavelet scale (thick continuous line) for finding start and end points. Maxima and minima related to the MUAP for the start and the end (thick crosses). (d) MUAP duration. Onset and offset (vertical lines) are shown and also the GSP (crosses) for this MUAP.

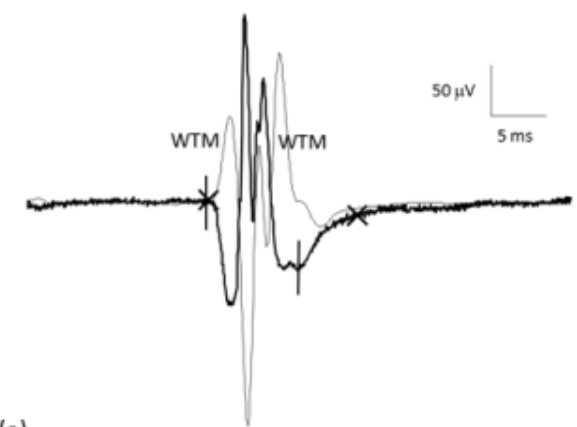

(a)

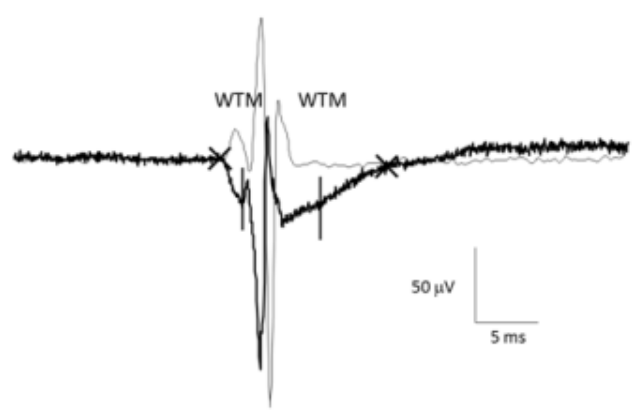

(b)

Figure 12. Errors in WTM start position in MUAPs with a small turn in their final (a) or initial part (b). Error in WTM in the end position in the low-slope tail of a MUAP in its terminal part (b). The waveform of the MUAPs (thick black line) and their selected scales of the DWT (thin grey line) are shown. GSP are in crosses. 
DWT-based automatic duration marker positions were compared with the corresponding manual positions for a small set of repeatedly recorded MUAPs. While no significant differences were found for the start point, the dispersion of automatic endpoint placements was lower than the dispersion of the corresponding manual placements. This points at the possibility of reaching more consistent estimates of this parameter with automatic procedures than with manual measurements.

\section{Conclusions and future perspectives}

The measurement of MUAP duration is a matter of particular difficulty. Especially difficult is placement of a MUAP's endpoint marker, and this is reflected in the high degree of variability observed in manual measurements of MUAP duration. Neither is the accuracy of automatic measurement of MUAP duration good, and thus continuous supervision and frequent manual revision of duration marker position are necessary. (Figure 13). Such manual adjustments are time consuming and tedious and still do not guarantee accuracy.

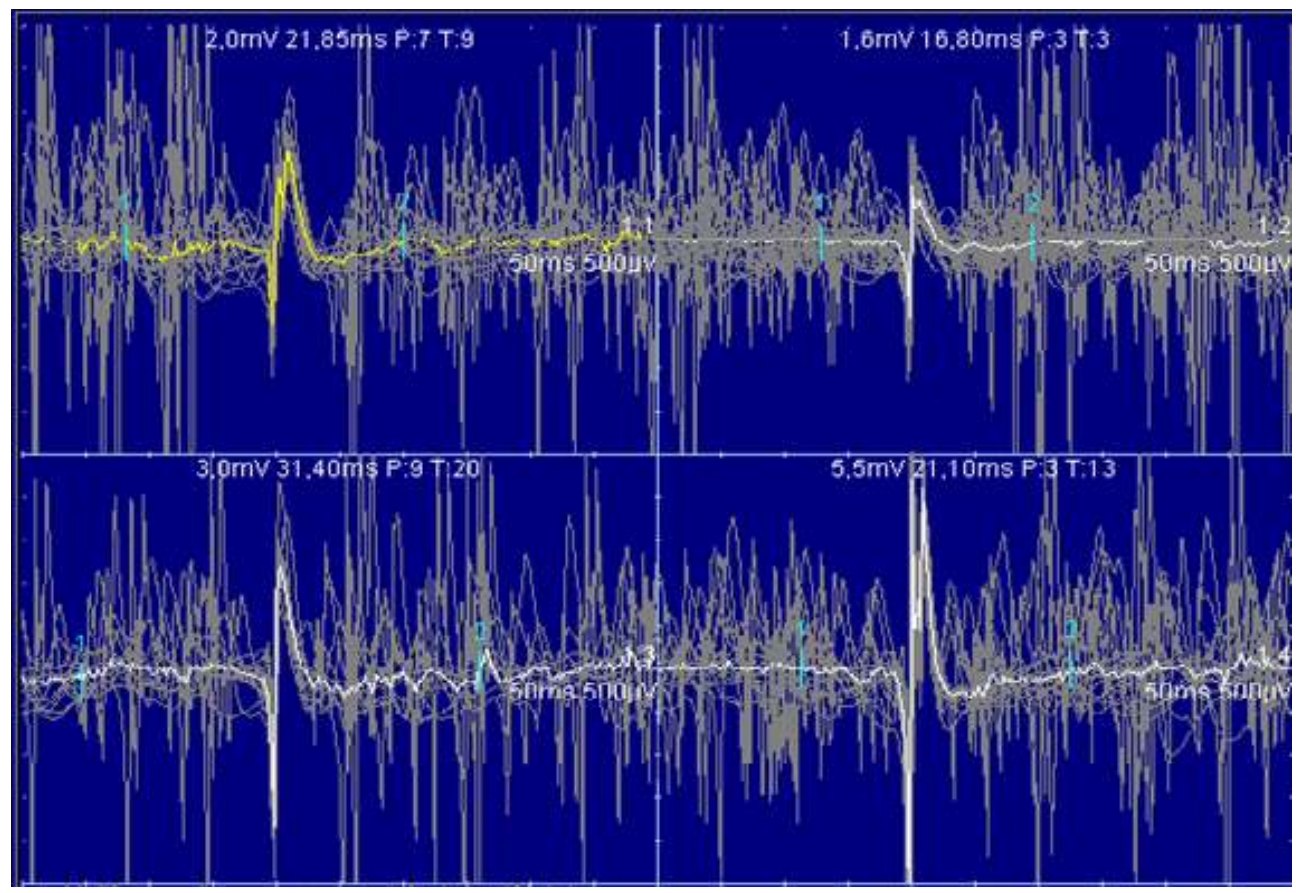

Figure 13. MUAPs automatically extracted by commercial equipment. MUAP durations are erroneously calculated and therefore manual corrections are needed.

Given the intrinsic difficulties, the measurement of MUAP duration has been described, quite correctly, as "an arbitrary task" [57]. However, the measurement of MUAP duration cannot be bypassed or avoided: not only does duration provide physiological information 
about the MU, it also delimits the MUAP waveform within which other MUAP parameters are measured. Thus, measurement of MUAP duration is an essential issue in EMG examinations, and it is of necessity the first task that must be accomplished before determination of other MUAP features.

Since we must have a measurement of MUAP duration, there is a strong requirement for a method which can provide "acceptable" estimations. By "acceptable" we recognize that there is not a unique true value of clinical duration. As has been discussed above, manual measurement does not ensure consistent estimates but there is reason to hope that an automatic method could be consistent enough. An automatic method might be considered good if it never makes gross misplacements, demonstrates low variability, works in real time and can deal with the relatively noisy signals found in daily clinical practice. An automatic method will show maximum repeatability because it will always give the same positions markers on re-analysis of a given MUAP input signal. If an effective automatic method suffers from any bias in marker positioning, it will be systematic and homogeneous in trend and magnitude, not arbitrary as occurs with subjective manual placements. Thus, the ideal method for attaining satisfactory consistency in MUAP duration measurement is an automatic method, which will overcome the inherent variability of human assessment.

The new, DWT-based computational strategy described above has demonstrated clear improvement in performance relative to conventional algorithms. There is, however, still significant room for betterment. More important than the results per se is the indication that some of the seemingly intractable difficulties in the management of bioelectrical recordings can be successfully overcome by new technologies of signal processing. The relevance of this conclusion extends beyond the area of EMG studies: the problems related to noise and variability in MU recording and measurement procedures are present in all the modalities of neurophysiologic studies and in Electromedicine in general. The measurement of MUAP duration is illustrative of the problematic nature of the analysis of bioelectric signals, but can be approached and managed with the latest signal processing techniques. Indeed, these techniques are being applied to other EMG features [58], such as the study of muscular fatigue [59], decomposition of surface EMG recordings [60, 61] and noise reduction for MUAPs extraction [62].

With respect to MUAP duration, further theoretical and empirical research is needed to develop automatic methods to provide robust and objective measurements, so that the MUAP duration measurement ceases to be an arbitrary task. Accurate and reliable automatic measurement of MUAP duration running on commercial equipment will serve to reduce the requirement for manual intervention in duration marker placement thereby helping the electromyographist. Together with multi-MUAP systems, automatic measurement methods could also contribute to a reduction in patient discomfort by shortening the examination time. Moreover, the availability of robust duration measurements would provide data of sufficient consistency and comparability for input into expert systems for diagnostic purposes, a natural goal of medical technology in the 21st Century. 


\section{Author details}

Ignacio Rodríguez-Carreño

Universidad de Navarra, Department of Quantitative Methods in Economics, Pamplona, Spain

Luis Gila-Useros

Complejo Hospitalario de Navarra, Clinical Neurophysiology Service, Pamplona, Spain

Armando Malanda-Trigueros

Universidad Pública de Navarra, Department of Electrical and Electronic Engineering, Pamplona, Spain

\section{References}

[1] Kimura J (2001). Electrodiagnosis in diseases of nerve and muscle: principles and practice. 3rd edition. New York: Oxford University Press.

[2] Dumitru D, Amato AA, Zwarts M. (2002). Electrodiagnostic medicine. 2nd edition. Hanley Belfus, Philadelphia,

[3] Stalberg E, Nandedkar S, Sanders DB, Falck B (1996). Quantitative motor unit potential analysis. J Clin Neurophysiol. 13: 401-422.

[4] Katz B (1966). Nerve, muscle, and synapse. McGraw-Hill, New York,

[5] Feinstein B, Lindegard B, Nyman E, Wohlfart G (1955). Morphologic studies of motor units in normal human muscles. Acta Anat; 23: 127-142.

[6] Nandedkar S, Sanders D, Stalberg E (1985). Selectivity of electromyography recording techniques: a simulation study. Med Biol Eng Comp; 23: 536-540.

[7] Nandedkar S, Sanders D, Stalberg E, Andreassen S (1988). Simulation of concentric needle EMG motor unit action potentials. Muscle Nerve; 11: 151-159.

[8] Stalberg E, Trontelj J (1994). Single fiber electromyography in healthy and diseased muscle. 2nd edition. Raven Press, New York, 291 p.

[9] Nandedkar S, Barkhaus P, Sanders D, Stalberg E (1988). Analysis of the amplitude and area of the concentric needle EMG motor unit action potentials. Electroencephalogr Clin Neurophysiol; 69: 561-567.

[10] Sonoo M, Stalberg E (1993). The ability of PAUM parameters to discriminate between normal and neurogenic PAUMs in concentric EMG: analysis of the PAUM "thickness" and the proposal of "size index". Electroencephalogr Clin Neurophysiol; 89: 291-303.

[11] Stewart C, Nandedkar S, Massey J, Gilchrist J, Barkhaus P, Sanders D (1989). Evaluation of an automatic method of measuring features of motor unit action potentials. Muscle Nerve; 12: 141-148.

[12] Zalewska E, Hausmanowa-Petrusewicz I (1995). Evaluation of MUAP shape irregularity - a new concept of quantification. IEEE Trans Biomed Eng; 42: 616-620.

[13] Zalewska E, Rowinska-Marcinska K, Hausmanowa-Petrusevicz I (1998). Shape irregularity of motor unit potentials in some neuromuscular disorders. Muscle Nerve; 21: 1181-1187. 
[14] Stalberg E, Sonoo M (1994). Assessment of variability in the shape of the motor unit action potential, the "jiggle," at consecutive discharges. Muscle Nerve; 17: 1135-1144.

[15] Campos C, Malanda A, Gila L, Segura V, Lasanta MI, Artieda J (2000). Quantification of jiggle in real electromyographic signals. Muscle Nerve; 23: 1022-1034.

[16] Stalberg E, Andreassen S, Falck B, Lang H, Rosenfalck A, Trojaborg W (1986). Quantitative analysis of individual motor unit potentials - a proposition for standardized terminology and criteria for measurement. J Clin Neurophysiol; 3: 313348.

[17] Lang A, Tuomola H (1974). The time parameter of motor unit potentials recorded with multi-electrode and the summation technique. Electromyogr Clin Neurophysiol; 14: 513-525.

[18] Buchthal F, Guld C, Rosenfalck P (1954). Action potential parameters in normal muscle and their dependence on physical variables. Acta Physiol Scand; 32: 200.

[19] Thiele B, Boehle A (1978). Anzahl der Spike-Komponenten im Motor Unit Potential. EEG-EMG-Zeitung Elektroenzephogr Vervandte Geb;9:125-130

[20] Lang A, Partanen J (1976). "Satellite potentials" and the duration of potentials in normal, neuropathic and myopathic muscles. J Neurol Sci; 27: 513-524.

[21] Kosarov D, Gydikov A (1970). The influence of volume conduction on the shape of action potentials recorded by various types of needle electrodes in normal human muscles. Electromyogr Clin Neurophysiol; 333: 319-325.

[22] Chu J, Chan RC, Bruyninckx F (1986). Effects of the EMG amplifier settings on the motor unit action potential parameters recorded with concentric and monopolar needles. Electromyogr Clin Neurophysiol; 26: 627-639.

[23] Lang A, Vaahtoranta K (1973). The baseline, the time characteristics and the slow afterwaves of the motor unit potential. Electroencephalogr Clin Neurophysiol; 35: 387394.

[24] Ma JA, Liveson DM (1992). Laboratory reference of clinical neurophysiology. F.A. Davis, Philadelphia.

[25] Buchthal F, Rosenfalck P (1955). Action potential parameters in different human muscles. Acta Psychiatr Scand; 30: 25-131.

[26] Bischoff C, Stalberg E, Falck B, Edebol Eeg-Olofsson K (1994). Reference values of motor unit action potentials obtained with multi-MUAP analysis. Muscle Nerve; 17: 842-851.

[27] Howard J, McGill K, Dorfman L (1988). Age effects on properties of motor unit action potentials: ADEMG analysis. Ann Neurol; 24: 207-213.

[28] Dorfman L, Robinson L (1997). AAEM Minimonograph 47: normative data in electrodiagnostic medicine. Muscle Nerve; 20: 4-14.

[29] Dumitru D, King JC (1999). Motor unit action potential duration and muscle lenght. Muscle Nerve; 22: 1188-1195.

[30] Dumitru D, King JC, Zwarts MJ (1999). Determinants of motor unit action potential duration. Clin Neurophysiol; 110: 1876-1882. 
[31] Lateva Z, McGill K (1998). The physiological origin of the slow afterwave in muscle action potentials. Electroencephalogr Clin Neurophysiol; 109: 462-469.

[32] Bromberg MB, Smith AG, Bauerle J (1999). A comparison of two commercial quantitative electromyographic algorithms with manual analysis. Muscle Nerve; 22: 1244-1248.

[33] Pfeiffer G, Kunze K (1992). Turn and phase counts of individual motor unit potentials: correlation and reliability. Electroencephalogr Clin Neurophysiol; 85: 161-165.

[34] Stalberg E, Falck B, Sonoo M, Astrom M (1995). Multi-MUP EMG analysis-a two year experience with a quantitative method in daily routine. Electroencephalogr Clin Neurophysiol; 97: 145-154.

[35] Nandedkar S, Barkhaus P, Charles A (1995). Multi-motor unit action potential analysis (MMA). Muscle Nerve; 18: 1155-1166.

[36] Nirkko AC, Rösler KM, Hess CW (1995). Sensitivity and specificity of needle electromyography: a prospective study comparing automated interference pattern analysis with single motor unit potential analysis. Electroencephalogr Clin Neurophysiol; 97: 1-10.

[37] Takehara I, Chu J, Li TC, Schwartz I (2004). Reliability of quantitative motor unit action potential parameters. Muscle Nerve; 30: 111-113.

[38] Chu J, Takehara I, Li TC, Schwartz I (2003). Skill and selection bias has least influence on motor unit action potential firing rate/frequency. Electromyogr Clin Neurophysiol; 43: 387-392.

[39] Nandedkar S, Barkhaus P, Sanders D, Stalberg E (1988). Analysis of the amplitude and area of the concentric needle EMG motor unit action potentials. Electroencephalogr Clin Neurophysiol; 69: 561-567.

[40] Montgomery DC, Runger GC (1993). Gage capability and designed experiments. Part I: Basic methods. Quality Engineering; 6. pp. 115-135.

[41] Montgomery DC, Runger GC (1993). Gage capability and designed experiments. Part II: Experimental design models and variance component estimation. Quality Engineering; 6. pp 289-305.

[42] Rodríguez I, Gila L, Malanda A, Gurtubay I, Mallor F, Gómez S, Navallas J, Rodríguez J (2007). Motor unit action potential duration, I: variability of manual and automatic measurements. J Clin Neurophysiol; 24: 52-58.

[43] Automotive Industry Action Group (AIAG), (2002). Measurement systems analysis. 3rd edition.

[44] Takehara I, Chu J, Schwartz I, Aye HH (2004). Motor unit action potencial (MUAP) parameters affected by editing duration cursors. Electromyogr Clin Neurophysiol; 44: 265-269.

[45] Rodríguez I, Gila L, Malanda A, Gurtubay IG, Navallas J, Rodríguez J (2010). Application of a novel automatic duration method measurement based on the wavelet transform on pathological motor unit action potentials Clin Neurophysiol; 121: 15741583. 
[46] Rodríguez I, Malanda A, Gila L, Navallas J, Rodríguez Falces J. Filter design for cancellation of baseline-fluctuation in needle EMG recordings (2006). Comput Methods Programs Biomed; 81: 79-93.

[47] Alvarez I, Rodríguez I, Gila L, Malanda A, Navallas J, Rodríguez J (2006). Influence of baseline fluctuation cancellation on automatic measurement of the motor unit action potential duration. Clin Neurophysiol; 117 (Suppl 1): S77.

[48] Akay M (1996). Detection and estimation methods of biomedical signals. Academic Press, New York,.

[49] Kumaran MS, Devasahayam SR, Sreedhar T (2000). Wavelet decomposition of the blik reflex R2 component enables improved discrimination of multiple sclerosis. Clin Neurophysiol; 111: 810-820.

[50] Fang J, Agarwall GC, Shahani BT (1999). Decomposition of multiunit electromyographic signals. IEEE Trans Biomed Eng; 46: 685-697.

[51] al-Fahoum AS, Howitt I (1999). Combined wavelet transformation and radial basis neural networks for classifying life-threatening cardiac arrhythmias. Med Biol Eng Comput; 37: 566-573.

[52] Cuiwei L, Chongxun Z, Changfen T (1995). Detection of ECG characteristic points using wavelet transforms. IEEE Trans Biomed Eng; 42: 21-28.

[53] Geva AB, Kerem DH (1998). Forecasting generalized epileptic seizures from the EEG signal by wavelet analysis and dynamic unsupervised fuzzy clustering. IEEE Trans Biomed Eng; 45: 1205-121.

[54] Gurtubay IG, Alegre M, Labarga A, Malanda A, Iriarte J, Artieda J (2001). Gamma band activity in an auditory oddball paradigm studied with the wavelet transform. Clin Neurophysiol; 112: 1219-1228.

[55] Rodríguez I, Gila L, Malanda A, Gurtubay I, Mallor F, Gómez S, Navallas J, Rodríguez J (2007). Motor unit action potential duration, II: a new automatic measurement method based on the wavelet transform. J Clin Neurophysiol; 24: 59-69.

[56] Goldberg DE (1989). Genetic algorithms in search, optimization and machine learning. Reading: Addison-Wesley,

[57] Sonoo M (2002). New attempts to quantify concentric needle electromyography. Muscle Nerve; Suppl 11: S98-S102.

[58] Raez MBI, Hussain MS, Mohd-Yasin F (2006). Techniques of EMG signal analysis: detection, processing, classification and applications. Biol Proced Online; 8: 11-35.

[59] Kumar DK, Pah ND, Bradley A (2003). Wavelet analysis of surface electromyography to determine muscle fatigue. IEEE Trans Neural Syst Rehabil Eng; 11: 400-406.

[60] Yamada R, Ushiba J, Tomita Y, Masakado Y (2003). Decomposition of electromyographic signal by principal component analysis of wavelet coefficient. IEEE EMBS Asian-Pacific Conference on Biomedical Engineering. Keihanna,: 118-119.

[61] Wimalaratna HS, Tooley MA, Churchill E, Preece AW, Morgan HM (2002). Quantitative surface EMG in the Diagnosis of neuromuscular disorders. Electromyogr Clin Neurophysiol; 42: 167-174. 
[62] Ren X, Yan Z, Wang Z, Hu X (2006). Noise reduction based on ICA decomposition and wavelet transform for the extraction of motor unit action potentials. J Neurosci Methods; 158: 313-322. 